\title{
Comment to "Weak Instruments Robust tests in GMM and the New Keynesian Phillips curve" by Frank Kleibergen and Sophocles Mavroeidis *
}

\author{
Fabio Canova \\ ICREA-UPF, AMeN and CEPR
}

This version: January 2009

\begin{abstract}
I discuss the identifiability of a structural New Keynesian Phillips curve when it is embedded in a small scale dynamic stochastic general equilibrium model. Identification problems emerge because not all the structural parameters are recoverable from the semi-structural ones and because the objective functions I consider are poorly behaved. The solution and the moment mappings are responsible for the problems.
\end{abstract}

JEL classification numbers: C10, C52, E32, E50

Key words: Identification, DSGE models, New Keynesian Phillips curve, Identification robust estimation methods.

\section{Introduction}

Kleinbergen and Mavroeidis (KM) have written an excellent paper, compactly reviewing what we know about the identification of the parameters of a New Keynesian Phillips curve when estimated by GMM, and contributed with interesting Monte Carlo evidence to shed light on the properties of various identification-robust methods proposed in the literature. This comment takes on two issues of interest for applied macroeconomists that the paper has left on the back burner: nowadays structural, rather than semistructural Phillips curves of the type KM consider, are typically considered; for policy

${ }^{*}$ The financial support of the Spanish Ministry of Education through the grant SEJ-2004-21682-E and of the Barcelona Economic Program (CREA) is gratefully acknowledged. 
exercises, a Phillips curve is typically embedded into a small or medium scale general equilibrium (DSGE) model. Therefore, the identification of its parameters requires a system-wide rather than a single equation perspective.

To discuss these issues I will first write down a canonical small scale structural model, which constitutes the backbone of those medium scale models currently used in policy institutions for forecasting and policy evaluation. I will then discuss the difference between the structural and the semi-structural versions of such a model and examine identification of the parameters when impulse responses or likelihood based methods are used to construct the objective function.

I want to stress that this comment is concerned with population identification problems. That is, the problems I highlight are intrinsic to the theory rather than specific to a data set or a sample. Their solutions therefore require alterations of the theory rather than the acquisition of better or longer data sets and/or a careful selection of objective functions to be optimized.

\section{A prototype small scale New Keynesian model}

The baseline model I consider has log-linearized optimality conditions of the form:

$$
\begin{aligned}
y_{t} & =\frac{h}{1+h} y_{t-1}+\frac{1}{1+h} E_{t} y_{t+1}+\frac{1}{\phi}\left(i_{t}-E_{t} \pi_{t+1}\right)+v_{1 t} \\
\pi_{t} & =\frac{\omega}{1+\omega \beta} \pi_{t-1}+\frac{\beta}{1+\omega \beta} E_{t} \pi_{t+1}+\frac{(\phi+\nu)(1-\zeta \beta)(1-\zeta)}{(1+\omega \beta) \zeta} y_{t}+v_{2 t} \\
i_{t} & =\lambda_{r} i_{t-1}+\left(1-\lambda_{r}\right)\left(\lambda_{\pi} \pi_{t-1}+\lambda_{y} y_{t-1}\right)+v_{3 t}
\end{aligned}
$$

where $h$ is the degree of habit persistence, $\phi$ the relative risk aversion coefficient, $\beta$ the discount factor, $\omega$ the degree of price indexation, $\zeta$ the degree of price stickiness, $\nu$ the elasticity of labor supply, while $\lambda_{r}, \lambda_{\pi}, \lambda_{y}$ are monetary policy parameters. $v_{1 t}$ and $v_{2 t}$ are $\operatorname{AR}(1)$ processes with parameters $\rho_{1}, \rho_{2}$, while $v_{3 t}$ is iid. The variances of the shocks are denoted by $\sigma_{i}^{2}, i=1,2,3$. Equation (1) is a log-linearized Euler condition; the second is a version of a New Keynesian Phillips curve obtained by log-linearizing the optimal pricing decision around a zero steady state inflation; and the third is a policy rule. The model has 14 structural parameters: $\theta_{1}=\left(h, \phi, \beta, \omega, \nu, \zeta, \lambda_{r}, \lambda_{\pi}, \lambda_{y}\right)$ are economic parameters and $\theta_{2}=\left(\sigma_{1}^{2}, \sigma_{2}^{2}, \sigma_{3}^{2}, \rho_{1}, \rho_{2}\right)$ are auxiliary parameters. While the specification is rather standard, two features of (1)-(3) are worth discussing. First, the policy rule is backward looking - this allows to name $v_{3 t}$ a monetary policy innovation. 
Second, there is habit in consumption, a feature typically absent from basic versions of the theory, but always included in the larger scale structures.

The semi-structural version of the model eschews the cross-equation restrictions that the theory imposes on the coefficients and is of the form:

$$
\begin{aligned}
y_{t} & =a_{1} y_{t-1}+a_{2} E_{t} y_{t+1}+a_{3}\left(i_{t}-E_{t} \pi_{t+1}\right)+v_{1 t} \\
\pi_{t} & =a_{4} \pi_{t-1}+a_{5} E_{t} \pi_{t+1}+a_{6} y_{t}+v_{2 t} \\
i_{t} & =a_{7} i_{t-1}+a_{8} \pi_{t-1}+a_{9} y_{t-1}+v_{3 t}
\end{aligned}
$$

Note that (5) corresponds to the specification used by KM. This version of the model also has 14 parameters, $\alpha=\left(a_{1}, \ldots, a_{9}\right)$ and $\theta_{2}=\left(\sigma_{1}^{2}, \sigma_{2}^{2}, \sigma_{3}^{2}, \rho_{1}, \rho_{2}\right)$ but following the logic of rank and order conditions, one can see that even when all the parameters of (4)-(6) were identifiable, it is impossible to recover all the $\theta_{1}$ from estimates of the $a^{\prime}$ s - $\zeta$ and $\nu$ enter multiplicatively and only in the slope parameter $a_{6}$, while $a_{1}$ and $a_{2}$ contain information only about $h$. Hence, conditioning on a model where variables are expressed in deviation from the steady state, and absent external information, it will be in general impossible to examine, e.g., the structural determinants of the slope of the Phillips curve and, as a consequence, back out estimates of the frequency of price adjustments, $\zeta$. Clearly, to solve this problem, it is necessary to specify additional equations which allow the elasticity of labor supply $\nu$ to be identifiable - for example, one could solve the model around a flexible price equilibrium, rather than the steady state, and add to the system of equations the definition of flexible output.

\section{Mapping the semi-structural model into a population objective function}

Local identification of the parameters of the model (4)-(6) requires that the objective function has a unique extremum in correspondence of the true parameter vector; that the Hessian of the objective function is of full rank in the neighborhood of the true parameter vector; and that the curvature of the objective function in the neighborhood of the true parameter vector is sufficient to translate the objective function information into parameters information.

Absent the first condition, models with different theoretical features may be observationally equivalent, given a particular objective function (see Sargent (1978), Kennan (1988), Kim (2001), Neely, et. al. (2001), Beyer and Farmer (2004), Canova and 
Sala (2006) among others). Clearly, observational equivalence crucially depends on the selected objective function. The second condition ensures that under-identification pathologies where the objective function is insensitive to variations in one or more parameters (see Choi and Phillips (1992) and Canova and Sala (2006)) will be absent. For example, the belief that the discount factor $\beta$ is hard to estimate with cyclical data in a Real Business Cycle model can be formalized by showing that the rank of the Hessian of the objective function is deficient for any true $\beta \in[0.96,0.9999]$.

The first two conditions rule out somewhat extreme kinds of identification pathologies. The third safeguards against more subtle weak and partial-identification problems. Deficiencies in the curvature of the objective function in the neighborhood of the true parameter vector in fact imply that parameter changes only marginally affect the objective function - it is either nearly flat in some dimensions (weak identification) or displays ridges (partial-identification).

The mapping from the parameters of the model (4)-(6) to a given objective function may fail to meet these three necessary criteria for identification because three types of transformations are needed to go from the former to the latter. First, the model needs to be solved - this involves a non-linear and typically numerical transformation. Second, some sufficient statistic (unconditional moments or impulse responses) is computed to summarize the informational content of the solution - this is another non-linear transformation. Third, an objective function expressing the distance between model-based and actual summary statistics is constructed - this can be a highly non-linear transformation if, e.g., one compares turning points of economic activity. When likelihood based methods are used, the last two steps are convoluted into one and the $\operatorname{VAR}(1)$ solution directly used to construct the likelihood or the kernel of the posterior. When some variables appearing in the solution are omitted because, e.g., they are unobservable, the solution for the observables is an $\operatorname{ARMA}(\infty, \infty)$ (see Canova (2007)) so one extra non-linear transformation is needed.

It is difficult to study in theory how these non-linear transformations repackage the information contained in the parameters. However, one can use graphical and exploratory analyses to detect problems. To compare my conclusions with those of KM, I will solely focus attention on the identification of $a_{4}, a_{5}$ and $a_{6}$, which give us information about the structural parameters $\beta, \omega$ and, given estimates of $a_{3}$, about the conglomerate of $\zeta$ and $\nu$. To make the discussion concrete, I choose the true parameter vector $\theta$ to be $\beta=0.985, \phi=2.0, \nu=1.0, \zeta=0.68, \omega=0.70, h=0.85, \rho_{r}=$ 
$0.2, \rho_{y}=1.1, \rho_{\pi}=1.5, \rho_{1}=0.65, \rho_{2}=0.65, \sigma_{1}=0.003, \sigma_{2}=0.002, \sigma_{3}=0.001$, in line with Rabanal and Rubio-Ramirez's (2005) estimates. These values imply that $a_{4}=0.4143, a_{5}=0.5830, a_{6}=0.2759$ are the true values of the parameters of interest.

I consider three objective functions: one measures the distance of responses to monetary policy shocks - twenty equally weighted responses of the three variables are used; the second is the likelihood function, constructed under normality of the disturbances; the third the kernel of the posterior, obtained using informative priors for the structural parameters entering $a_{4}, a_{5}$ and $a_{6}$, centered at the true values with small spreads.

\section{Are the parameters of the Phillips curve theoretically identifiable?}

For this class of models and for my choice of "true" $\theta$, all the objective functions have a unique local extremum. Five of the eigenvalues of the Hessian of the distance function are exactly zero - those corresponding to $\rho_{1}$ and $\rho_{2}$, which are underidentified from monetary policy shocks, and those corresponding to $\sigma_{i}, i=1,2,3$, which are underidentified from any scaled impulse responses. The other two objective functions have no eigenvalue with this feature. Nevertheless, six eigenvalues of both the Hessian of the distance function and of the likelihood function are small relative to the average eigenvalue - weak and partial identification problems could be present. To examine if these eigenvalues are associated with $a_{4}, a_{5}$ and $a_{6}$, I graphically explore how the objective functions change when these parameters vary in a neighborhood of the true parameter vector (see the range presented in the x-axis in Figure 1), keeping all other parameters are fixed at their true values.

The distance function is rather flat in all dimensions (the elasticity is always smaller than 0.1) and somewhat asymmetric in $a_{4}$. When plotted in two dimensions, it is still very flat particularly for $a_{5}$, the forward looking parameter of the Phillips curve. The log likelihood function, which contains all the information of the model, is better behaved except for the marked asymmetry it displays in all dimensions. When plotted in two dimensions it has sufficient curvature in both $a_{4}, a_{5}$ and $a_{5}, a_{6}$ but displays diagonal ridges $-a_{4}, a_{5}$ and $a_{6}$ are not separately identifiable. The log posterior kernel, instead, is nicely peaked in all dimensions. Since the priors used in structural estimation are conventionally centered at calibrated values and with tight spreads - as we have done here - it is not difficult to see that the prior may determine the shape of the posterior. 

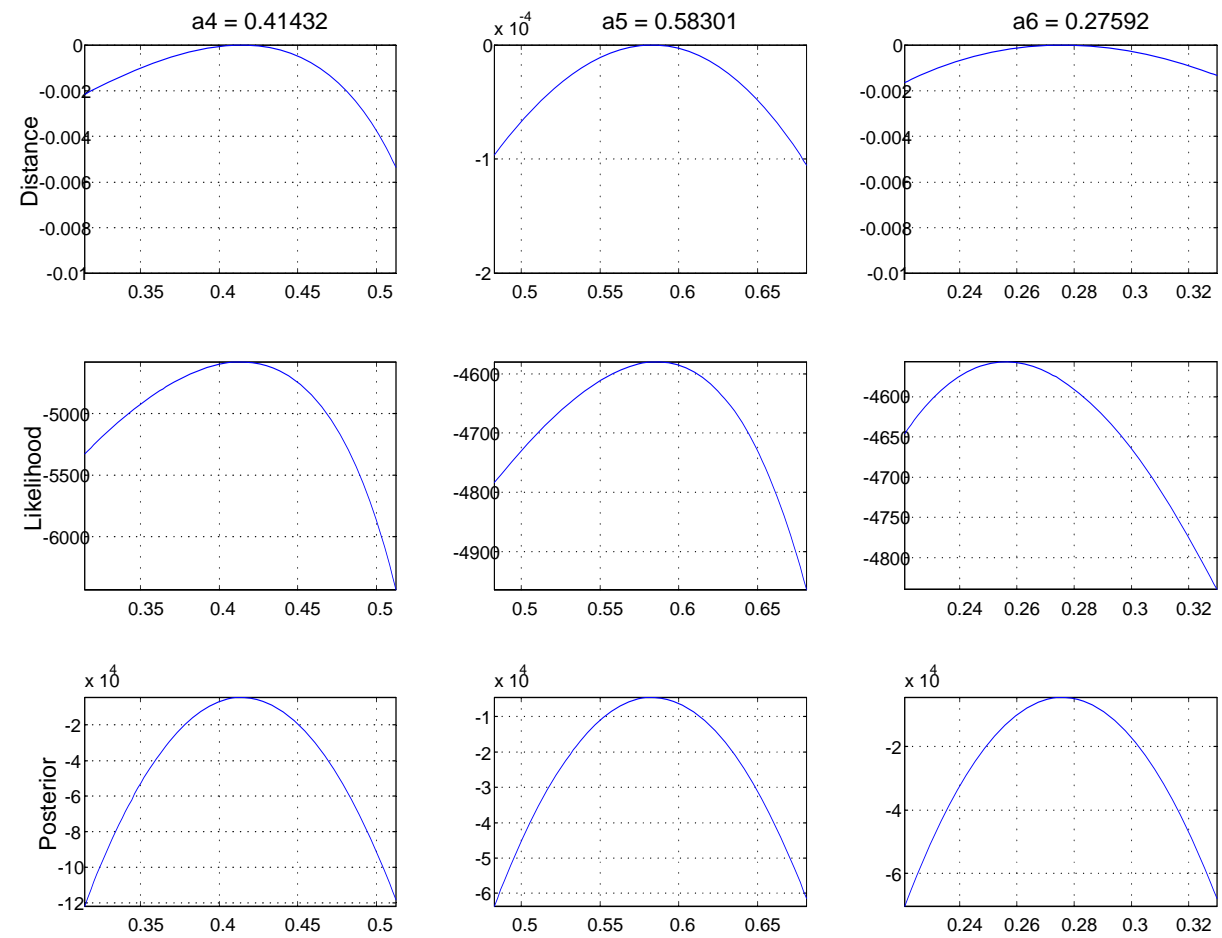

Figure 1: Shape of different objective functions

These visual impressions are confirmed using the relative size of the eigenvalues of the Hessian of the objective function at the true parameters. For example, the eigenvalues of the Hessian of the distance associated with $a_{4}$ and $a_{6}$ are of the order of ten percent of the average eigenvalue of both matrices, and the one associated with $a_{5}$ is smaller than 0.001 percent of the average eigenvalue.

In conclusion, both the distance and the likelihood functions will have hard time to appropriately identify the forward looking parameter of the Phillips curve but for different reasons: distance function because identification of $a_{5}$ is weak; the likelihood function because $a_{5}$ is linearly related to the other parameters of the Phillips curve.

Which mapping is responsible for these information deficiencies? The solution and moment mappings both contribute. In the solution mapping four of the nine eigenvalues are smaller than 0.20 of the average eigenvalue, while with the moment mapping two additional eigenvalues are smaller than 0.20 of the average. Since the smallest eigenvalue of the solution mapping is the one associated with $a_{5}$, identification of this parameter is 
difficult unless the model or the way it is solved is changed. Note that the use of higher order approximations does not guarantee better identification properties in population (see e.g. Canova and Sala (2006)).

\section{Estimation}

Since it is unlikely that applied investigators will spend time altering the theory or refining their numerical solution techniques, estimation methods that work when the identification problems exist are needed. While KM have made it clear that identification robust methods exist in the single equation GMM literature, no procedure has been devised for likelihood based methods. Furthermore, while impulse response matching estimators share similarities with GMM, failure to use the continuously updating weighting matrix in the estimation precludes a direct extension of the GMM results.

In this section, I first show what identification problems imply when non-identification robust methods are used to estimate the parameters of the Phillips curve and then I used ideas of the literature KM review to construct estimates of the parameters of interest. The punchline is the following: when weak and partial identification problems are present, standard methods produce erratic estimates and meaningless standard errors, even in extremely large samples. However, estimation intervals obtained inverting the objective function are practically identical to those obtained with standard methods because the distance function is extremely flat in many dimensions (compare with Nason and Smith (2008)). This is perhaps unsurprising since the distance function I use is not a robust objective function in the sense of KM.

The exercise is as follows. Given the correct model and 500 initial conditions in the neighborhood of the true parameter vector, I estimated $a_{4}, a_{5}$ and $a_{6}$ using a distance function which measures how far output gap, inflation and the nominal rate responses to monetary shocks in the model are from the true ones. Figures 2 and 3 present the histograms of initial and final estimates for two different choices of the true parameter vector; the $\mathrm{x}$-axis shows the range for the chosen initial conditions. When the forward looking component of the Phillips curve is strong and the slope economically different from zero, problems are concentrated in $a_{5}$ - the range of final estimates of $a_{5}$ is only marginally smaller than the range of initial conditions, and the correlation between initial conditions and final estimates is high (around 0.7). 

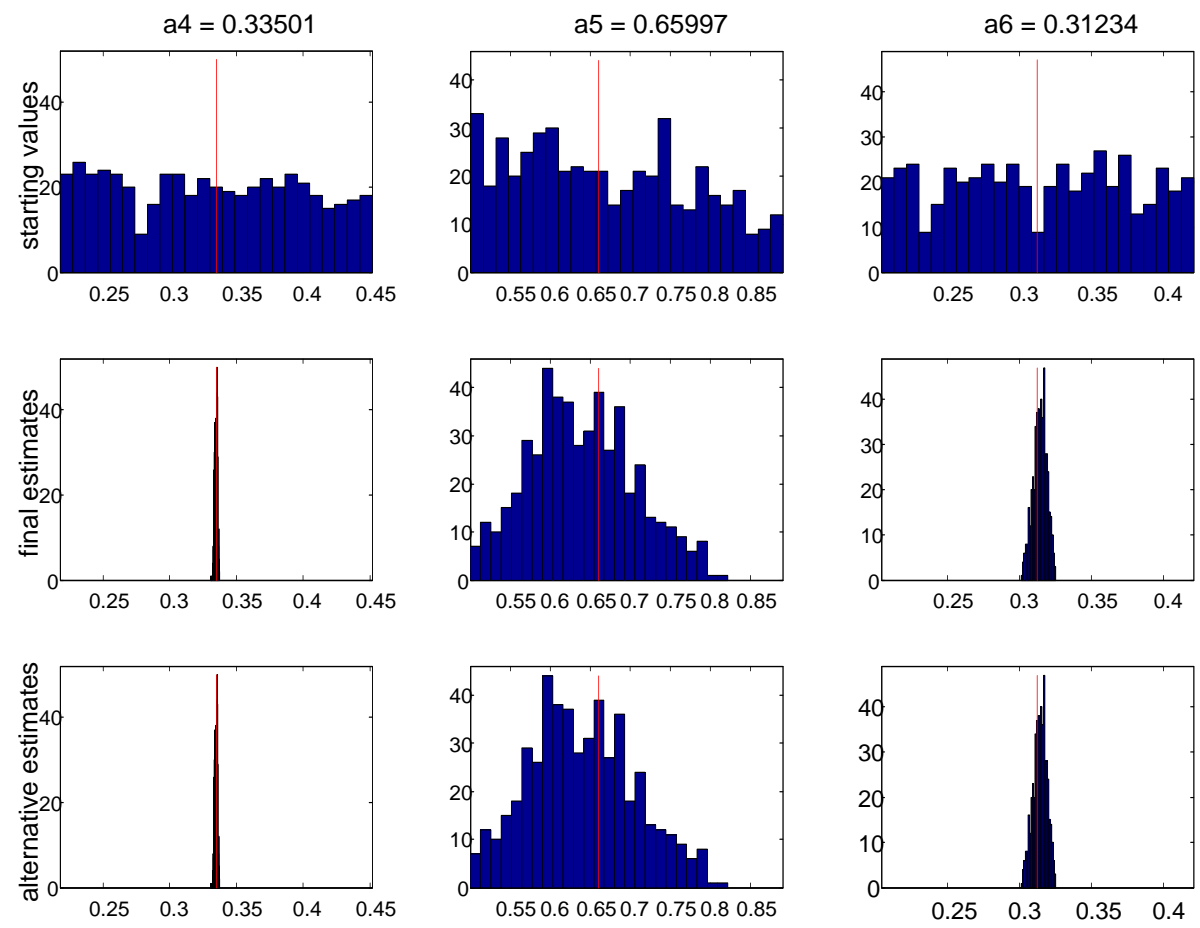

Figure 2: Histogram of intial conditions and estimates

When the forward and the backward looking components are roughly similar and marginal costs are important for inflation, estimates of all semi-structural parameters are always away from the true parameters, the sum of estimates of $a_{4}$ and $a_{5}$ always exceeds one, the slope of the Phillips curve systematically underestimated, and the bias large (order of 10-25 percent). When sample rather than population objective functions are available, all these problems could be greatly magnified.

Figures 2 and 3 shows that the range of estimates of $a_{5}$ obtained by inverting the objective function is practically identical to the one obtained with standard minimum distance estimators - out of the 500 cases only five are eliminated. This is because, in all the simulations I have run, the value of the objective function at the estimates is close to the median value of the $\chi^{2}(51)$ distribution. This could have been expected: figure 1 the objective function is so flat in $a_{5}$ that estimates in the range $[0.45,0.80]$ only very marginally change its value. 

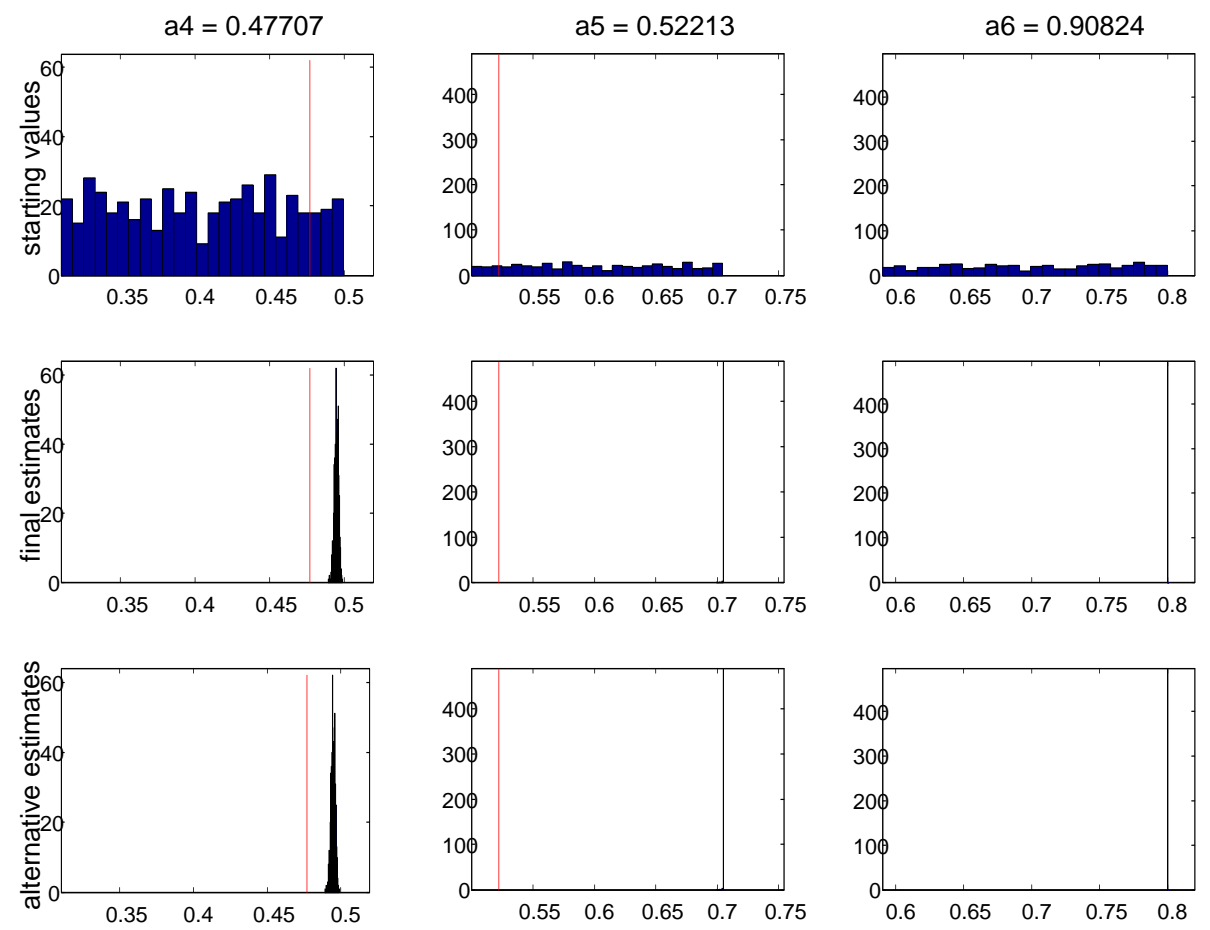

Figure 3: Histogram of intial conditions and estimates

To conclude, the problems that KM highlighted in their excellent review get compounded when the New Keynesian Phillips curve is embedded into a small scale DSGE model and multivariate estimation techniques are considered (see also Cochrane (2007)); in addition there are additional headaches for applied investigators when structural, rather than semi-structural, estimation is attempted. The solution mapping seems to be responsible for the identification difficulties. Poorly behaved solution mappings are especially problematic because they leave applied investigators with no choice other than respecify the structure they wish to estimate or refine their solution procedure.

\section{References}

[1] Beyer, A. and Farmer, R. (2004) On the indeterminacy of new Keynesian Economics, ECB working paper 323.

[2] Canova, F. (2007) Methods for Applied Macroeconomic Research, Princeton University Press. 
[3] Canova, F. and Sala, L. (2006) Back to square one: identification issues in DSGE models, ECB working paper 583.

[4] Canova, F. and Sala, L. (2008) Back to square one: identification issues in DSGE models, revision, available at www.crei.cat/people/canova, forthcoming Journal of Monetary Economics

[5] Choi, I and P.C. Phillips (1992) Asymptotic and Finite Sample Distribution Theory for IV Estimators and Tests in Partially Identified Structural Equations, Journal of Econometrics 51, 113-150.

[6] Cochrane, J. (2007) Identification and price determination with Taylor rules: A critical review, University of Chicago, manuscript.

[7] Kennan, J. (1998) An econometric analysis of the fluctuations in aggregate labor supply and demand, Econometrica, 45, 969-990.

[8] Kim, J. (2003) Functional equivalence between intertemporal and multisectoral investment adjustment costs, Journal of Economic Dynamics and Control, 27, 533-549.

[9] Nason J. and Smith G: W. (2008), Identifying the new Keynesian Phillips curve, Journal of Applied Econometrics, 23, 525-551.

[10] Neely, C., Roy, A., Whiteman, C. (2001) Risk Aversion versus Intertemporal Substitution: A case study of identification failures in the Intertemporal Capital Asset Pricing Model, Journal of Business and Economic Statistics, 19, 295-403.

[11] Rabanal, P. and Rubio-Ramirez, J. (2005) Comparing New-Keynesian Models of the Business Cycle: A Bayesian Approach, Journal of Monetary Economics, 52, 1150-1162.

[12] Sargent, T. (1978) Estimation of Dynamic Labor Demand Schedules under Rational Expectations, Journal of Political Economy, 86, 1009-1044. 